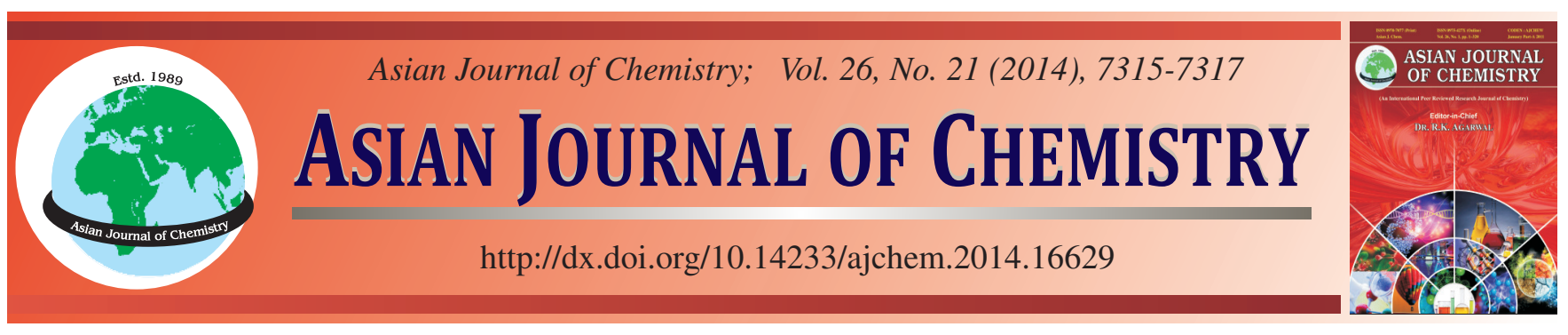

\title{
Effect of Aluminium Content on the Structural, Acidic and Catalytic Properties of Mesoporous Aluminosilicate Materials
}

\author{
Ling Xu*, Xuxia Bao, Maoping Lv, Limei Duan and Zongrui Liu
}

College of Chemistry and Chemical Engineering, Inner Mongolia University for Nationalities, Tongliao 028043, P.R. China

*Corresponding author: E-mail: tlxuling1979@163.com; jdxuling1979@aliyun.com

A series of mesoporous aluminosilicate materials with different Si/Al molar ratio was synthesized using citric acid as pore forming agent. The effect of varied aluminium content on the structural and acidic properties of the mesoporous aluminosilicate materials was characterized through $\mathrm{N}_{2}$ adsorption-desorption, ${ }^{27} \mathrm{Al}-\mathrm{MAS}$ NMR and $\mathrm{NH}_{3}$-TPD techniques. The results showed that the structural and acidic performances of the mesoporous aluminosilicate materials were strongly related to the aluminium content. Furthermore, the catalytic activity of the mesoporous aluminosilicate materials with different $\mathrm{n}_{\mathrm{si}} / \mathrm{n}_{\mathrm{Al}}$ ratio was studied by the alkylation of phenol with tert-butanol. Both the catalytic activity and the selectivity to major product 2,4-di-tert-butyl phenol increased with the addition of aluminium content from Si/Al molar ratio of 50 to 10 .

Keywords: Mesoporous material, Aluminium content, Catalytic property.

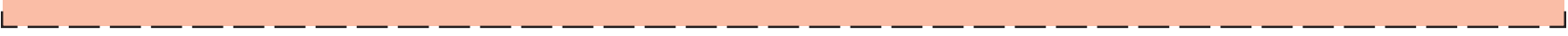

\section{INTRODUCTION}

During the last decade, mesoporous materials have attracted much attention because of their high surface areas and large pore channels. They have interesting applications in catalysis, catalytic supports, adsorbents and drug delivery, etc $^{1-3}$. Generally, the mesoporous silica materials are synthesized by coope-rative formation mechanism. Notably, MCM-41 and SBA-15 have been prepared using cetyltrimethyl ammonium bromide and triblock copolymers poly(ethylene oxide)-poly(alkylene oxide)-poly(ethylene oxide) as structure directing agents and tetraethoxysilane as silica source in basic and acidic medium, respectively ${ }^{4,5}$. The preparation process of mesoporous materials is very easy, whereas it suffers from the drawback of the expensive and toxic organic compound as template. The economic and environmental considerations have recently initiated an interest in the use of inexpensive and green substance as structure directing agent.

It is well known that organic additives such as D-glucose, D-maltose, hydroxyl-carboxylic acid and citric acid etc. are environmentally friendly and non-toxic organic compounds. Furthermore, they have the ability to aggregate and can be used as pore forming agent. Takahashi et al. ${ }^{6}$ and Wei et al. ${ }^{7}$ have reported the synthesized method of mesoporous silica materials using above-mentioned organic additives as poreforming agent. However, they found that it was difficult to induce $\mathrm{Al}$ atom into the silica framework, when citric acid (CA) was used as pore forming agent. The materials consisting of pure silica are of limited use for catalytic application due to the lack of catalytically active sites.

Recently, we have studied the incorporation of $\mathrm{Al}$ atom into the mesoporous silica materials based on the fact that the citric acid can react with aluminium species to form CA-Al complexes ${ }^{8}$. In order to investigate the structural and catalytic performances of the mesoporous aluminosilicate materials, we synthesized a series of the mesoporous aluminosilicate materials using citric acid as pore forming agent, which varies the aluminium content in the initial sol-gel.

\section{EXPERIMENTAL}

The mesoporous aluminosilicate materials were synthesized with hydrothermal crystalline $\operatorname{method}^{8}$. The initial aluminosilicate precursor solution was prepared with the molar composition of $0.5 \mathrm{Al}_{2} \mathrm{O}_{3}: \chi \mathrm{SiO}_{2}: 4 \mathrm{TPAOH}: 60 \mathrm{C}_{2} \mathrm{H}_{5} \mathrm{OH}$ : $750 \mathrm{H}_{2} \mathrm{O}$, where $\chi=50,25$ and 10 . Then constant amount of citric acid was added into the above aluminosilicate precursor solution under stirring, respectively. The final gel was crystallized in a Teflon-lined autoclave at $373 \mathrm{~K}$ for 2 day. The crystallized product was filtered off, washed with deionized water, dried and calcined in air at $823 \mathrm{~K}$ for $5 \mathrm{~h}$. The calcined materials were designated as MM- $\chi$, where $\chi$ denotes the $\mathrm{Si} / \mathrm{Al}$ molar ratio of the reaction mixture. 


\section{RESULTS AND DISCUSSION}

$\mathbf{N}_{\mathbf{2}}$ adsorption-desorption analysis: The structural properties of the mesoporous aluminosilicate materials were investigated by $\mathrm{N}_{2}$ adsorption-desorption technique. The isotherms, corresponding pore szie distributions are shown in Fig. 1. In general, it can be seen that the mesoporous aluminosilicate materials exhibit isotherms of type IV with hysteresis loops of type $\mathrm{H}_{2}$, which is characteristic to mesoporous materials with "ink-bottle" type mesopores ${ }^{9}$. A sharp increase in the volume of $\mathrm{N}_{2}$ adsorbed is observed in all $\mathrm{N}_{2}$ isotherms above $\mathrm{P} / \mathrm{P}_{0}$ of 0.5 , which is ascribed to the capillary condensation step.

In addition, the uptake of hysteresis loops with the addition of aluminium content is shifted to higher relative pressures, which indicates that an increase in the pore size as compared to the materials having a lower aluminium contents. The pore size distribution curves in Fig. 1 (inset) are confirmed the result of the adsorption-desorption isotherms. Generally, the pore size distribution curves transfer broader with the addition of aluminium content from $\mathrm{n}_{\mathrm{Si}} / \mathrm{n}_{\mathrm{Al}}$ of 50 to 10 . In other words, the correspongding pore size distribution curve of the mesoporous material with $\mathrm{n}_{\mathrm{Si}} / \mathrm{n}_{\mathrm{Al}}=50$ shows relatively narrower pore size distribution compared to the material wiht $\mathrm{n}_{\mathrm{Si}} / \mathrm{n}_{\mathrm{Al}}=$ 10. The reason may be described by the fact that more citric acid molecules are needed to interact with increased aluminium species to form larger CA-Al complex and the space occupied with larger CA-Al remains as pores after decomposition of citric acid $^{7}$.

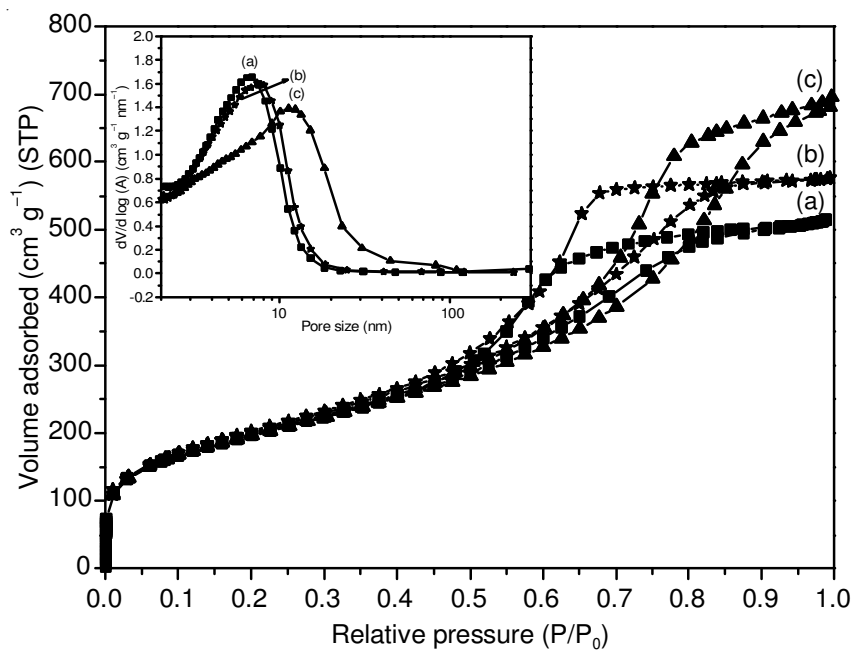

Fig. 1. $\quad \mathrm{N}_{2}$ adsorption-desorption isotherms and pore size distribution (inset) of the mesoporous materials: (a) MM-50, (b) MM-25 and (c) MM10

${ }^{27}$ Al-MAS NMR results: The mesoporous aluminosilicate materials were also studied by ${ }^{27} \mathrm{Al}-\mathrm{MAS}$ NMR to confirm the incorporation of $\mathrm{Al}$ atoms in the silica framework and to determine their coordination environment. The ${ }^{27} \mathrm{Al}-\mathrm{MAS}$ NMR spectra are shown in Fig. 3. The mesoporous aluminosilicate materials exhibit resonances at 53 and 0 ppm associated with tetrahedral and octahedral (non-framework) Al, respectively ${ }^{10}$. The result show that most of $\mathrm{Al}$ in mesoporous materials is tetrahedrally coordinated even at high aluminium content $\left(\mathrm{n}_{\mathrm{Si}} / \mathrm{n}_{\mathrm{Al}}=10\right)$. In addition, the tetrahedral to octahedral
Al ratio is found to increase with more aluminium incorporation as shown in Fig. 2 a to $2 \mathrm{c}$, which indicates that more $\mathrm{Al}$ atom has been incorporated into the silica framework with the addition of aluminium content in the initial solution.

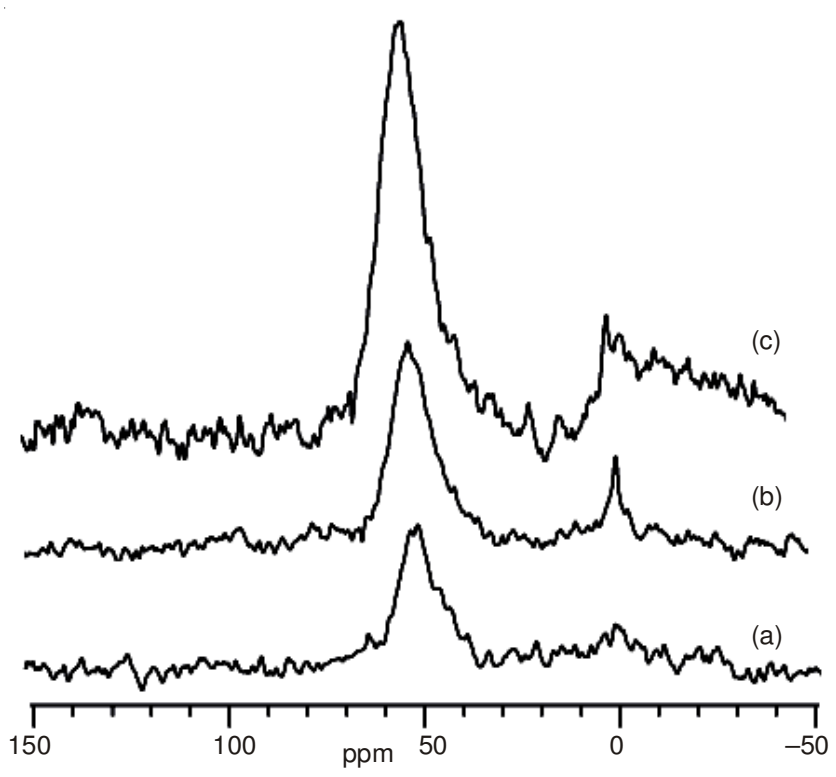

Fig. 2. ${ }^{27}$ Al-MAS NMR spectra of the mesoporous materials: (a) MM-50, (b) MM-25 and (c) MM-10

$\mathbf{N H}_{3}$-TPD analysis: The acid sites distribution of the mesoporous aluminosilicate materials was determined by $\mathrm{NH}_{3}^{-}$ TPD measurements and the $\mathrm{NH}_{3}$-TPD profiles are shown in Fig. 3. We can assume that the incorporation of $\mathrm{Al}$ gives rise to weak and strong acid sites due to the presence of two ammonia desorption peaks in the $\mathrm{NH}_{3}$-TPD profiles of the mesoporous aluminosilicate materials. The low temperature peak is ascribed to the weak acid sites and the high temperature peak is associated with the strong acid sites, respectively. Furthermore, the acidic strength and the density of acid sites increase with increased aluminium content from $\mathrm{Si} / \mathrm{Al}$ molar ratio of 50 to 10.

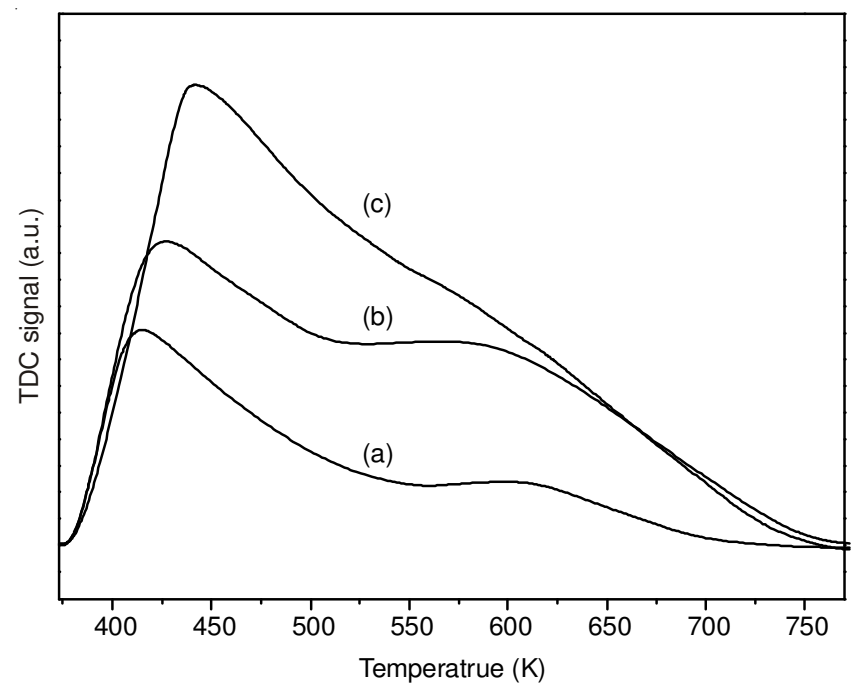

Fig. 3. $\mathrm{NH}_{3}$-TPD profiles of the mesoporous materials: (a) MM-50, (b) MM-25 and (c) MM-10 


\begin{tabular}{|c|c|c|c|c|c|c|}
\hline \multirow{3}{*}{ Catalysts } & \multicolumn{5}{|c|}{$\begin{array}{c}\text { TABLE-1 } \\
\text { CATALYTIC ACTIVITY AND PRODUCT DISTRIBUTION }\end{array}$} & \\
\hline & \multirow{2}{*}{$\begin{array}{l}\text { Conversion of } \\
\text { phenol }(\%)\end{array}$} & \multicolumn{5}{|c|}{ Selectivity of product (\%) } \\
\hline & & 2,6-DTBP & 2,4,6-ТTBP & 2-TBP & 4-ТВP & 2,4-DTBP \\
\hline MM-50 & 77.8 & - & 1.2 & 8.8 & 46.6 & 43.3 \\
\hline MM-25 & 92.3 & 0.1 & 1.1 & 3.7 & 50.5 & 44.7 \\
\hline MM-10 & 95.8 & 0.1 & 1.6 & 3.1 & 45.1 & 50.1 \\
\hline
\end{tabular}

Catalytic results: tert-Butylation of phenol is a kind of Friedel-Crafts alkylation and can be catalyzed by all kinds of acid sites. The reaction products such as 2-tert-butyl phenol (2-TBP), 4-tert-butyl phenol (4-TBP) and 2,4-di-tert-butyl phenol (2,4-di-TBP) are valuable intermediates in the production of a variety of chemicals and commercial products. It is well known that the catalytic activity and the product selectivity rely mainly on the density and the strength of acid sites. Besides, the porosity of material has significant effect on the catalytic behaviour of the material due to the large reactants, intermediate products and products involved in the reaction process. Accordingly, the mesoporous aluminosilicate materials possessed large pore size and strong acidity (confirmed by $\mathrm{N}_{2}$ adsorption-desorption and $\mathrm{NH}_{3}$-TPD results, respectively), were appropriate for catalyzing the alkylation of phenol with tertbutanol. The catalytic activity and product selectivity are listed in Table-1.

In the present study, the reaction between phenol and tertbutyl alcohol was performed in gas phase, under mild conditions: $\mathrm{T}=418 \mathrm{~K}, \mathrm{n}_{\text {Phenol }}: \mathrm{n}_{\text {TBA }}=1: 2.5$ (TBA corresponding to tert-butyl alcohol), WHSV $=2.20 \mathrm{~h}^{-1}$. In the case of MM50 with the low aluminium content, it exhibits low phenol conversion $(77.8 \%)$. With the addition of $\mathrm{Al}$ from $\mathrm{Si} / \mathrm{Al}$ molar ratio of 50 to 10 , the phenol conversion increases significantly to $95.8 \%$, which can be assigned to the increased density of acid sites. In common, the main product selectivity of 2,4-ditert-butyl phenol also increases from 43.3 to $50.1 \%$, with increased aluminium content from $\mathrm{Si} / \mathrm{Al}$ molar ratio of 50 to 10. The catalytic results imply that the pore size of catalysts large enough for the reaction, the density and strength of acid site play the decisive role in the tert-butylation of phenol.

\section{Conclusion}

In conclusion, we synthesized a series of mesoporous aluminosilicate materials and determined the effect of Si/Al molar ratio on the intrinsic properties of the mesoporous materials. The results showed that the pore volume, pore size, acidity and catalytic activity of the mesoporous aluminosilicate materials increased with the addition of aluminium content in the initial gel from $\mathrm{Si} / \mathrm{Al}$ molar ratio of 50 to 10 . Furthermore, the high catalytic activity of the mesoporous aluminosilicate catalysts and the selectivity to main product were ascribed to strong acidity resulted from the increased aluminium content in the framework.

\section{ACKNOWLEDGEMENTS}

The authors acknowledge the Program for Young Talents of Science and Technology in Universities of Inner Mongolia Autonomous Region (NJYT-12-B17), the Inner Mongolia Education Department (NJZY12121) and the financial support of the National Natural Science Foundation of China (No. 21261014).

\section{REFERENCES}

1. F. Wei, Z. Liu, J. Lu and Z.-T. Liu, Micropor. Mesopor. Mater., 131, 224 (2010)

2. X. Li, D. Han, H. Xue, X. Liu and Z. Yan, Int. J. Mater. Res., 102, 1473 (2011).

3. X. Gui, A. Xie, Y. Shen, Z. Pan and S. Sun, Int. J. Mater. Res., 102, 1493 (2011).

4. C.T. Kresge, M.E. Leonowicz, W.J. Roth, J.C. Vartuli and J.S. Beck, Nature, 359, 710 (1992).

5. D. Zhao, J. Feng, Q. Huo, N. Melosh, G.H. Fredrickson, B.F. Chmelka and G.D. Stucky, Science, 279, 548 (1998).

6. R. Takahashi, S. Sato, T. Sodesawa, M. Kawakita and K. Ogura, J. Phys. Chem. B, 104, 12184 (2000).

7. Y. Wei, D. Jin, T. Ding, W.-H. Shih, X. Liu, S.Z.D. Cheng and Q. Fu, Adv. Mater., 10, 313 (1998).

8. L. Xu, S. Wu, J. Guan, Y. Ma, K. Song, H. Xu, C. Xu, Z. Wang and Q. Kan, Catal. Commun., 9, 1970 (2008).

9. J.C. Groen, L.A.A. Peffer and J. Pérez-Ramírez, Micropor. Mesopor. Mater., 60, 1 (2003).

10. A.D. Irwin, J.S. Holmgren and J. Jonas, J. Non-Cryst. Solids, 101, 249 (1988). 